\title{
Fluid bilayer phase in aqueous mixtures of fatty alcohol and cationic surfactant
}

\author{
Tiago Espinosa de Oliveira $\odot$ \\ Institut Charles Sadron, CNRS and University of Strasbourg, Rue du Loess, F-67034 Strasbourg Cedex 2, France \\ and Universidade Federal do Rio Grande do Sul, Porto Alegre, Brazil \\ Fabien Leonforte, Luc Nicolas-Morgantini, Anne-Laure Fameau, and Bernard Querleux $\odot$ \\ L'Oréal Research and Innovation, F-93600 Aulnay-sous-Bois and F-93400 Saint-Ouen, France \\ Fabrice Thalmann $\odot$ and Carlos M. Marques $\odot$ \\ Institut Charles Sadron, CNRS and University of Strasbourg, Rue du Loess, F-67034 Strasbourg Cedex 2, France
}

(Received 17 July 2019; published 24 January 2020)

\begin{abstract}
Lamellar gel networks are creamy formulations extensively used in cosmetics and pharmaceutics. The basic units of these networks are lipid bilayers assembled from mixtures of fatty alcohols, surfactants, and water. Despite being an essential step for the preparation of the dispersions, the nature of the mixtures at high temperature has defied understanding. Here we show, by atomistic molecular dynamics simulations, that aqueous mixtures of cetyl $\left(\mathrm{C}_{16} \mathrm{OH}\right)$ and stearyl $\left(\mathrm{C}_{18} \mathrm{OH}\right)$ alcohols, and cetyl-trimethylammonium chloride (CTAC), lead to fluid bilayers, which are the units of the lamellar $L_{\alpha}$ phase. By providing a consistent numerical simulation model able to describe the structure and properties of fatty alcohol lamellae stabilized by a surfactant, our work paves the way for the elucidation of the forces regulating this family of industrially important gels.
\end{abstract}

DOI: 10.1103/PhysRevResearch.2.013075

Fatty alcohols (FAs) are amphiphilic molecules associating a polar hydroxyl head to an alkyl chain tail $\left[\mathrm{CH}_{3}-\left(\mathrm{CH}_{2}\right)_{n-1}-\mathrm{OH}\right]$ [1]. Cetyl $(n=16)$ and stearyl $(n=$ 18) alcohols, in combination with nonionic or ionic surfactants, are common ingredients in formulations of cosmetic and pharmaceutical cream products [2-7]. These aqueous dispersions, often known as lamellar gel networks, derive their advantageous properties from an extended and highly interconnected lamellar structure [8]. The basic network units are rigid $\left(L_{\beta}\right)$ bilayers, or stacks of bilayers, forming a percolating structure that can withstand elastic deformations [9-11].

Depending on temperature, fatty alcohols alone adopt various ordered structures with poor hydration capacity [12-14], preventing mixtures of water and fatty alcohols to be formulated as creams with the desired homogeneity, stability, and viscoelasticity: only when an appropriate proportion of surfactants is added to the mixture can the lamellar gel network structure be obtained [8].

The textural properties of the cream are empirically optimized by various preparation steps involving combinations of stirring and heating or cooling. But in all formulation variants, the different components are first brought together at high enough temperatures where a fluid bilayer $\left(L_{\alpha}\right)$ phase is believed to form [15]. Despite being a key determinant

Published by the American Physical Society under the terms of the Creative Commons Attribution 4.0 International license. Further distribution of this work must maintain attribution to the author(s) and the published article's title, journal citation, and DOI. for the formation of the lamellar network, the nature of such high-temperature phase of fatty alcohols/surfactant mixtures has not been thoroughly inspected. In particular, to our knowledge, there are no published molecular dynamics simulations of mixtures of water, fatty alcohol, and surfactants, thus precluding the long-sought elucidation of the forces shaping these membranes and controlling their interactions.

In this paper, we perform all-atom molecular dynamics simulations of aqueous solutions of cetyl $\left(\mathrm{C}_{16} \mathrm{OH}\right)$ and stearyl $\left(\mathrm{C}_{18} \mathrm{OH}\right)$ alcohols and cetyl-trimethylammonium chloride (CTAC), a typical mixture leading to the formation of lamellar gel networks [8,10,15]. Cetyl-trimethylammonium chloride (CTAC) is a cationic surfactant that mixes well with cetyl and stearyl alcohols that have similar chain lengths. We employ all-atom molecular dynamics simulations using the GROMACS 5.1.2 package [18]. We use the CHARMM36 force-field parameters [16,17] for $\mathrm{C}_{16} \mathrm{OH}, \mathrm{C}_{18} \mathrm{OH}$, and CTAC adapted from dioleoyl phosphatidyl choline (DPPC), a common glycerophospholipid, and methanol molecules. CHARMM-36 was shown to accurately describe the melting transition of DPPC lipid bilayers, as a result of some empirical optimization. Methanol $\mathrm{CH}_{3} \mathrm{OH}$ and DPPC phosphocholine parameters were used, respectively, to create the alcohol and trimethylammonium groups. The transferrable intermolecular potential with three points (TIP3P) water model was used for the solvent. All atomic types, charges, and interaction parameters $\sigma$ and $\epsilon$ used in the simulations are displayed in Table I.

All the systems were built using PACKMOL [19,20] and submitted to an energy-minimization step using the steepest descent algorithm [21], in order to relax the initial stresses. 
TABLE I. Standard atomic types, charges, and interaction parameters $(\sigma$ and $\epsilon)$ from the CHARMM-36 force field used in the simulations.

\begin{tabular}{|c|c|c|c|c|c|c|c|}
\hline \multicolumn{2}{|c|}{ Chain } & \multicolumn{2}{|c|}{ Atom } & \multirow{2}{*}{$\begin{array}{c}\text { Atom type } \\
\text { OG311 }\end{array}$} & \multirow{2}{*}{$\begin{array}{r}\text { Charge } \\
-0.650\end{array}$} & \multirow{2}{*}{$\begin{array}{c}\sigma(\mathrm{nm}) \\
0.315\end{array}$} & \multirow{2}{*}{$\frac{\epsilon(\mathrm{kJ} / \mathrm{mol})}{0.804}$} \\
\hline & & $\mathrm{OH}$ & $\mathrm{O}$ & & & & \\
\hline & & & $\mathrm{H}$ & HGP1 & +0.420 & 0.040 & 0.193 \\
\hline & FA & $\mathrm{CH}_{2}$ & $\mathrm{C}$ & CG321 & +0.050 & 0.358 & 0.234 \\
\hline & & & $\mathrm{H}$ & HGA2 & +0.090 & 0.239 & 0.146 \\
\hline \multirow[t]{5}{*}{ Head } & & $\mathrm{N}$ & $\mathrm{N}$ & NTL & -0.600 & 0.330 & 0.837 \\
\hline & & $\mathrm{CH}_{2}$ & $\mathrm{C}$ & CTL2 & -0.100 & 0.358 & 0.234 \\
\hline & CTAC & & $\mathrm{H}$ & HL & +0.250 & 0.125 & 0.192 \\
\hline & & $\mathrm{CH}_{3}$ & $\mathrm{C}$ & CTL5 & -0.350 & 0.367 & 0.335 \\
\hline & & & $\mathrm{H}$ & HL & +0.250 & 0.125 & 0.192 \\
\hline \multirow[t]{4}{*}{ Tail } & FA/CTAC & $\mathrm{CH}_{2}$ & $\mathrm{C}$ & CTL2 & -0.180 & 0.358 & 0.234 \\
\hline & & & $\mathrm{H}$ & HAL2 & +0.090 & 0.239 & 0.117 \\
\hline & & $\mathrm{CH}_{3}$ & $\mathrm{C}$ & CTL3 & -0.270 & 0.363 & 0.326 \\
\hline & & & $\mathrm{H}$ & HAL3 & +0.090 & 0.239 & 0.100 \\
\hline
\end{tabular}

The system is submitted to a short thermalization simulation (1 ns) using the Berendsen thermostat and barostat [22], with a coupling constant of $0.5 \mathrm{ps}$ at a temperature of $315 \mathrm{~K}$ and pressure 1 bar. The production simulations consisted of $100 \mathrm{~ns}$ runs with a varying temperature from 278 to $358 \mathrm{~K}$. The temperature was controlled by a Nosé-Hoover thermostat $[23,24]$ with a coupling constant of 0.5 ps. Simulations were performed with a constant pressure set to 1 bar using the Parrinello-Rahman [25] semi-isotropic barostat with a coupling time of $2.0 \mathrm{ps}$. The long-range electrostatic interactions were handled using the particle mesh Ewald (PME) method [26]. The interaction cutoff for nonbonded interactions was chosen equal to $1.2 \mathrm{~nm}$. The time step for the simulations was set to $2 \mathrm{fs}$ and the equations of motion were integrated using the leap-frog algorithm [27]. All H-bond vibrations were constrained using the LINCS algorithm [28].

Most of the simulated systems were made of 256 chains arranged into a symmetric bilayer conformation, each opposing leaflet containing 128 chains and solvated with 2560 water molecules. Initial configurations were prepared by molecular modeling. Four chemical compositions were considered: pure cetyl $\left(\mathrm{C}_{16} \mathrm{OH}\right), 20 \%$ molar surfactant $\left[\mathrm{C}_{16} \mathrm{OH}\right.$ : CTAC (4:1)], and equimolar cetyl-stearyl mixtures without $\left(\mathrm{C}_{16: 18} \mathrm{OH}\right)$ and with $20 \%$ molar $\left[\mathrm{C}_{16: 18} \mathrm{OH}\right.$ : CTAC (4:1)] surfactant. Triplicate $100 \mathrm{~ns}$ runs for annealing and quenching were performed to thermalize the systems. This choice for initial configurations was justified by a number of available experimental results consistent with a lowly hydrated gel $L_{\beta}$ structure at room temperature [15].

Structural changes in the FA organization were followed by computing the carbon-carbon order parameter $S$ of the alkyl chain with respect to the bilayer normal direction $z$. It is defined for every group $\left(\mathrm{CH}_{2}\right)_{i}$ in a chain as

$$
S_{i}=\frac{1}{2}\left\langle 3 \cos ^{2} \theta-1\right\rangle,
$$

where $\theta$ is the angle between the vector connecting the two nearest carbons surrounding the selected $\mathrm{CH}_{2}$ group and the membrane normal axis. Bilayer systems are characterized by a positive $S$, which is larger for gel than for fluid phases. Isotropic structures lead to a vanishing order parameter

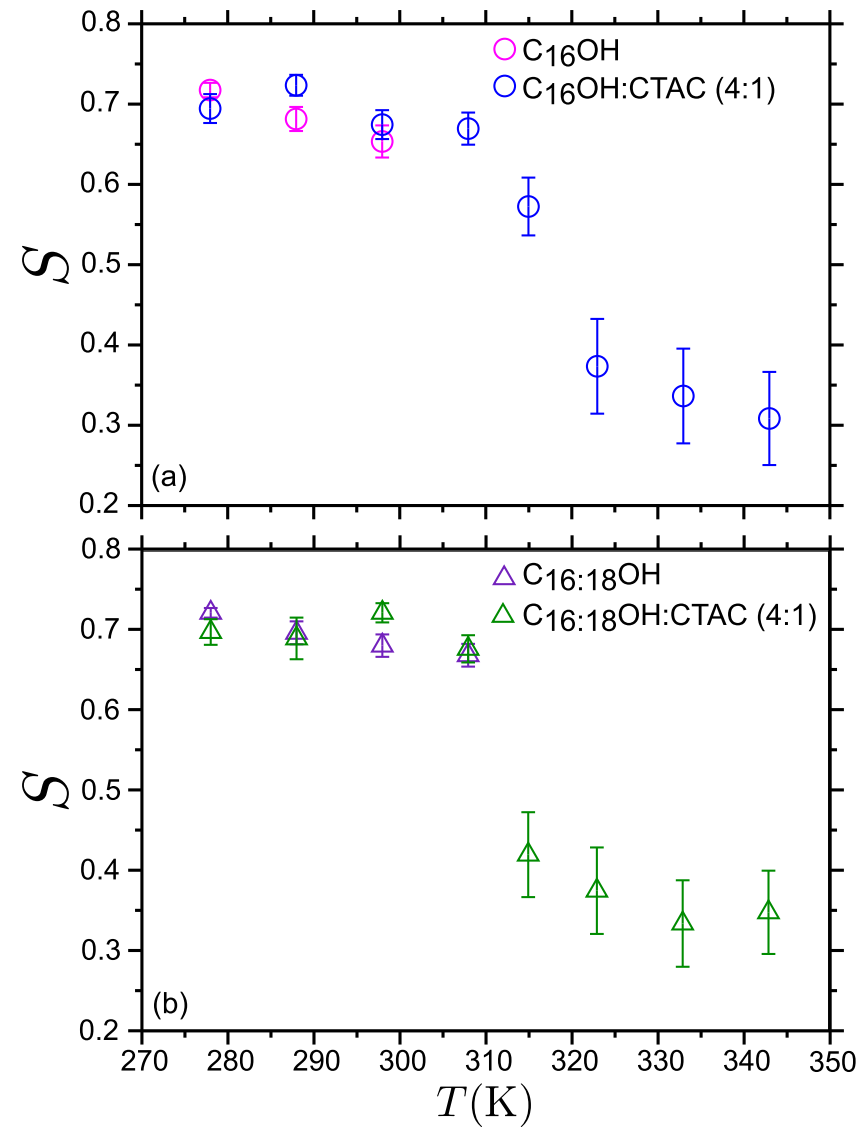

FIG. 1. Average order parameter as a function of temperature for four different compositions. (a) Aqueous $\mathrm{C}_{16} \mathrm{OH}$ and $\mathrm{C}_{16} \mathrm{OH}$ : CTAC (4:1) and (b) aqueous $\mathrm{C}_{16: 18} \mathrm{OH}$ and $\mathrm{C}_{16: 18} \mathrm{OH}$ : CTAC (4:1).

$S_{i}=0$. In all the cases of interest, the order parameter decreases as $i$ shifts from the FA head towards the terminal methyl group $\left(-\mathrm{CH}_{3}\right)$. An average order parameter $S$, representative from the central section of FA chains (averaged over 8 carbons, numbered from 4 to 11), was computed and represented in Fig. 1.

In the absence of surfactant, both $\mathrm{C}_{16} \mathrm{OH}$ and $\mathrm{C}_{16: 18} \mathrm{OH}$ showed a well-defined gel phase at temperatures below, respectively, 298 and $308 \mathrm{~K}$, with $S \sim 0.7$. In this phase, alkyl chains adopt well-packed dense all-trans conformations. At larger temperatures, these two systems were found in a completely disordered phase, presumably an isotropic fluid phase. The preferential arrangement of FA molecules into leaflets was lost. Cetyl-stearyl mixtures melted at slightly higher temperatures than pure cetyl systems, in agreement with experimental data [29].

In the presence of a 1:4 CTAC surfactant ratio (20\% of the system molar mass), a structure consistent with a lamellar fluid phase was observed in a 323-343 K temperature range. Tails were more disordered, i.e., melted, as shown by the lower value $S \sim 0.35$. Experimental systems of comparable FA-CTAC composition, but with much larger water contents, display a $L_{\beta} \rightarrow L_{\alpha}$ transition temperature at $243 \mathrm{~K}$. In our simulations, above $358 \mathrm{~K}$, an isotropic fluid phase similar to the one seen without CTAC prevailed. We observed that repeated cycles of annealing quenching resulted in a denser, 

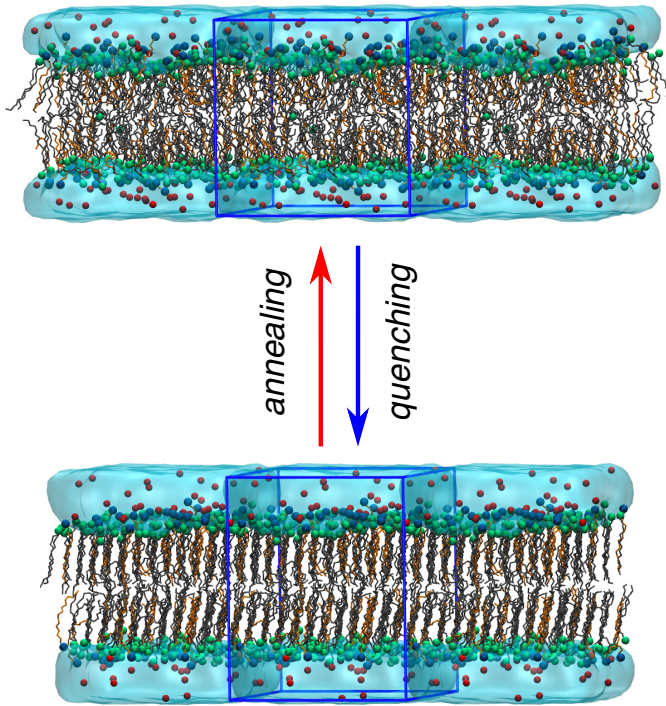

FIG. 2. Snapshots of the bilayer structures of the $\mathrm{C}_{16: 18} \mathrm{OH}$ : CTAC (4:1) fatty alcohol-surfactant mixture at low $\left(L_{\beta}\right)$ and high $\left(L_{\alpha}\right)$ temperature.

better packed, low-temperature solid structure, with an average order parameter higher than 0.7; see Fig. 2.

As further evidence of a lamellar fluid state stabilized by surfactants, we increased the size of the simulated systems and tested the ability of the bilayer to spontaneously form a vesicle, which is a topological hallmark of fluid bilayers. A large system comprising 2560 fatty chains was initially prepared as a flat bilayer. Then, a flat square slab was cut off and dunked into a large water reservoir, in such a way that the slab did not extend across periodic boundary conditions (PBCs) and had a free boundary line exposed to the solvent (Fig. 3 at $0 \mathrm{~ns}$ ). As the time sequence of snapshots displayed in Fig. 3 shows, the slab spontaneously closed on itself, adopting a small unilamellar vesicle shape. From 5 to $20 \mathrm{~ns}$, the bilayer formed a temporary bicelle disk, closed by a folded leaflet rim. This disklike structure remained for some time, while undergoing severe surface fluctuations. Then, it started to bend spontaneously after $30 \mathrm{~ns}$ and ended up forming the closed vesicle after $40 \mathrm{~ns}$ of simulation time. The vesicle stayed stable during the following $500 \mathrm{~ns}$ of simulation.

The lamellar fluid phase structure was characterized further by measuring the area per fatty chain $A_{f}$ and the membrane thickness $D_{m}$. Both parameters were found to evolve smoothly with temperature, except for an abrupt change between 315 and $323 \mathrm{~K}$, supporting evidence of a sharp gel-fluid transition (Table II). The bilayer isothermal stretching elastic modulus $K_{A}$ was estimated based on equilibrium box area fluctuations [30-34], according to the relation

$$
K_{A}=k_{B} T \frac{\langle A\rangle}{\left\langle A^{2}\right\rangle-\langle A\rangle^{2}},
$$

where $\langle A\rangle$ and $\left\langle A^{2}\right\rangle$ are the average value and the mean-square fluctuation (variance) of the sample monolayer area $A=$ $L_{x} L_{y}$, with $k_{B}$ the Boltzmann constant and $T$ the temperature. The area fluctuations arise from coupling the system to a semi-isotropic Parrinello-Rahman barostat [25] and a NoseHoover thermostat [23,24]. The Parrinello-Rahman barostat
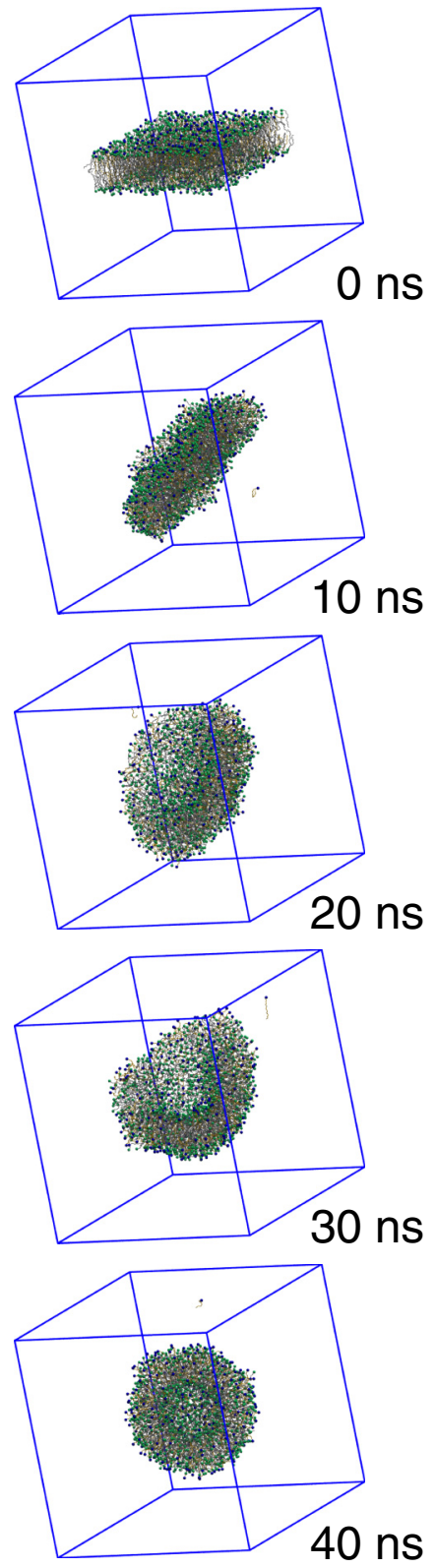

FIG. 3. Spontaneous formation of a small unilamellar vesicle from a $\mathrm{C}_{16: 18} \mathrm{OH}$ : CTAC (4:1) fatty alcohol-surfactant mixture at $333 \mathrm{~K}$.

is assumed to enforce a constant pressure and vanishing surface tension NP $\gamma \mathrm{T}$ ensemble, i.e., the system conformations contribute to the statistical average with a weight proportional to $\exp \left\{-\left(E+P L_{z} L_{x}^{2}\right) / k_{B} T\right\}$, with energy $E$, pressure $P$, and vanishing $\gamma$. This is achieved by allowing the $L_{z}$ and $L_{x}=L_{y}$ lateral box sizes to be independently rescaled. We found a significant drop in $K_{A}$ at the transition (Table II), consistent with a lamellar fluid $L_{\alpha}$ phase, with $K_{A}$ values comparable to those of lipid bilayers $(\sim 250 \mathrm{mN} / \mathrm{m})$ [35]. Note that imposing a semi-isotropic barostat is only possible if the simulated system possesses a finite stretching modulus $K_{A}$. The highesttemperature states occurring in the presence and in the absence of CTAC were shown to be unstable under a semiisotropic barostat, displaying unbound $L_{x}-L_{z}$ fluctuations, as expected from isotropic fluid phases. 
TABLE II. Geometrical parameters for gel and fluid bilayers of $\mathrm{C}_{16} \mathrm{OH}$ : CTAC (4:1): $A_{f}$ means cross-sectional area per chain and $D_{m}$ is the bilayer thickness. The relative statistical uncertainty for $A_{f}$ and $D_{m}$ is about 0.003 . The bilayer elastic coefficient is $K_{A}$, from a fluctuation analysis argument. The relative statistical uncertainty turns out to be larger, of the order of $20 \%$ to $30 \%$. All three parameters change sharply between 315 and $323 \mathrm{~K}$. Parameters of the bilayer are measured over the last $80 \mathrm{~ns}$ of $100 \mathrm{~ns}$ simulations.

\begin{tabular}{lccc}
\hline \hline$T(\mathrm{~K})$ & $A_{f}\left(\mathrm{~nm}^{2}\right)$ & $D_{m}(\mathrm{~nm})$ & $K_{A}(\mathrm{mN} / \mathrm{m})$ \\
\hline 278 & 0.23 & 4.07 & 2328.4 \\
288 & 0.23 & 4.08 & 2274.1 \\
298 & 0.23 & 4.09 & 2146.3 \\
308 & 0.23 & 4.10 & 1875.4 \\
315 & 0.23 & 4.20 & 2077.2 \\
323 & 0.29 & 3.49 & 216.9 \\
333 & 0.30 & 3.47 & 226.9 \\
343 & 0.31 & 3.43 & 173.9 \\
\hline \hline
\end{tabular}

Gel-fluid bilayer transitions are commonly believed to be weakly first order [36]. To gain insight into the thermodynamic transition properties, we estimated the change in enthalpy $\Delta H$ upon melting. The enthalpy of the bilayer and water system $H$ was defined as $H=U+P V$ [37], where $U$ is the time-averaged total energy, computed from the sum of all the potential energies in the force field and the translation kinetic energy of the atoms, $P$ is the target pressure of the barostat, $\gamma=0$, and $V$ is the average simulated volume. Bond vibrations are either treated classically or frozen in the case of hydrogen covalent bonds (methyl, hydroxyl, and water groups). Constrained bonds decrease the number of effective independent degrees of freedom and correspondingly reduce the kinetic-energy term. Within the limit of the force-field accuracy (e.g., truncation of van der Waals interactions, classical or frozen bond vibrations), the discontinuous change in internal energy and enthalpy should account fairly for the variation in cohesion, internal isomerization, and hydration energy contributions, which are expected to predominantly contribute to the transition.

Figure 4 represents the enthalpy difference $H(T)-$ $H(278 \mathrm{~K})$ as a function of temperature for $\mathrm{C}_{16} \mathrm{OH}: \mathrm{CTAC}$ (4:1) and $\mathrm{C}_{16: 18} \mathrm{OH}$ : CTAC (4:1) aqueous solutions. The enthalpy curve shows a smooth trend (constant pressure specific heat), except in the region of the melting transition. The enthalpy variation at the transition of $\mathrm{C}_{16} \mathrm{OH}$ : CTAC (4:1) and $\mathrm{C}_{16: 18} \mathrm{OH}$ : CTAC (4:1) was estimated by extrapolating the linear trends of the low- and high-temperature phases. We found, respectively, for these jumps, a value of 10.30 and $10.00 \mathrm{~kJ} / \mathrm{mol}$. This result is quantitatively consistent with experimental differential scanning calorimetry (DSC) data [15], and falls within the expected range of chain melting values for equivalent phospholipid systems [38]. We found that the surfactant containing cetyl and cetyl-stearyl mixtures melts at different temperatures, with the longer chain alcohol driving $L_{\beta} \rightarrow L_{\alpha}$ melting to higher temperatures, albeit with comparable transition enthalpies.

To summarize, we have proposed an atomistic model that successfully describes the cetyl and cetyl-stearyl alcohols' mixtures in aqueous solution with and without surfactants

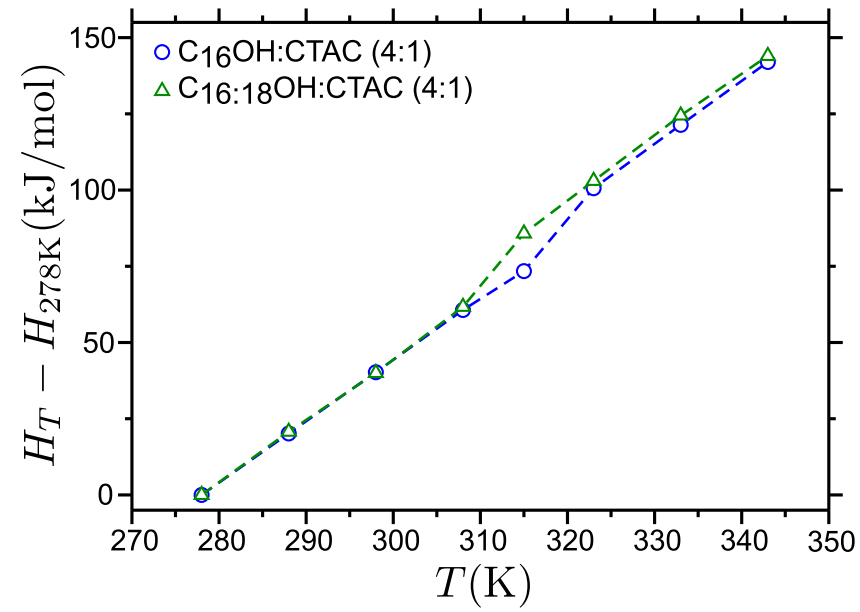

FIG. 4. Enthalpy as a function of temperature for the $\mathrm{C}_{16} \mathrm{OH}$ : CTAC (4:1) and $\mathrm{C}_{16: 18} \mathrm{OH}$ : CTAC (4:1) systems.

(CTAC). We investigated the structural, mechanical, and thermodynamic properties of these systems at various temperatures. In the absence of surfactant, we found a transition between a solid gel bilayer and an isotropic fluid phase, in good agreement with experiments. In the presence of surfactant (20\% CTAC), we found two transitions. A first transition was seen between a gel phase and fluid lamellar phase. Our estimate of the enthalpy of melting is very satisfactory, although, perhaps not surprisingly, our temperature transition is lower than that experimentally measured in more hydrated multilamellar systems [15]. We also found that the compressibility modulus falls abruptly upon melting to a value comparable to similar known $L_{\alpha}$ phases. The parameters used in the present work well describe the systems under investigation, both from the thermodynamic and from the mechanic point of view, and we confidently associate the lamellar fluid phase to a $L_{\alpha}$ phase, while the low-temperature gel phase is consistent with a $L_{\beta}$ state. Simulation of larger system sizes showed that the fluid bilayer may form stable unilamellar vesicles.

This work is a step toward a better assessment of the influence of the high-temperature phase of mixtures of fatty alcohols and surfactants on the formation of lamellar gel networks. We anticipate that it will allow, for instance, the exploration of the role of the surfactant molar fraction in the stabilization of the fluid bilayer phase, and the drawing of phase diagrams that can be directly compared to experiments. Importantly, the force-field model presented here paves the road for extending molecular dynamics (MD) simulations into multibilayer systems, where one can test the influence of the bilayer-bilayer interactions on the buildup of the lamellar network.

The authors acknowledge partial support from the Investissements d'Avenir program "Développement de l'Économie Numérique" through the SMICE project. T.E.d.O., F.T., and C.M.M. acknowledge partial support from L'Oréal Research \& Innovation, and F.L., L.N.-M., A.-L.F., and B.Q. are employees of L'Oréal Research \& Innovation. We acknowledge support from the HPC cluster TGCC (Très Grand Centre de Calcul du CEA, Bruyères-le-Châtel, France). 
[1] K. Noweck and W. Grafahrend, Ullmann's Encyclopedia of Industrial Chemistry (Wiley, New York, 2000).

[2] B. Barry, J. Colloid Interface Sci. 28, 82 (1968).

[3] G. Eccleston, J. Colloid Interface Sci. 57, 66 (1976).

[4] S. Fukushima, M. Takahashi, and M. Yamaguchi, J. Colloid Interface Sci. 57, 201 (1976).

[5] R. Rowe and J. McMahon, Colloids Surf. 27, 367 (1987).

[6] R. J. Goetz and M. S. El-Aasser, Langmuir 6, 132 (1990).

[7] T. S. Awad, E. S. Johnson, A. Bureiko, and U. Olsson, J. Dispersion Sci. Technol. 32, 807 (2011).

[8] T. Iwata, in Cosmetic Science and Technology: Theoretical Principles and Applications, edited by K. Sakamoto, R. Lochhead, H. Maibach, and Y. Yamashita (Elsevier, New York, 2017).

[9] W. J. Benton, C. A. Miller, and R. L. Wells, J. Am. Oil Chem. Soc. 64, 424 (1987)

[10] G. Eccleston, M. Behan-Martin, G. Jones, and E. TownsAndrews, Int. J. Pharm. 203, 127 (2000).

[11] T. Iwata and K. Aramaki, IFSCC Mag. 16, 249 (2013).

[12] K. Tanaka, T. Seto, and T. Hayashida, Bull. Inst. Chem. Res. 35, 123 (1958).

[13] K. Tanaka, T. Seto, A. Watanabe, and T. Hayashida, Bull. Inst. Chem. Res. 37, 281 (1959).

[14] S. Fukushima, M. Yamaguchi, and F. Harusawa, J. Colloid Interface Sci. 59, 159 (1977).

[15] K. Wunsch, P. Relkin, G. Cuvelier, F. Clément, L. NicolasMorgantini, H. Benkhelifa, and D. Flick, J. Therm. Anal. Calorim. 123, 1411 (2016).

[16] A. D. Mackerell, J. Comput. Chem. 25, 1584 (2004).

[17] J. B. Klauda, R. M. Venable, J. A. Freites, J. W. O`Connor, D. J. Tobias, C. Mondragon-Ramirez, I. Vorobyov, A. D. MacKerell, and R. W. Pastor, J. Phys. Chem. B 114, 7830 (2010).

[18] M. Abraham, D. van der Spoel, E. Lindahl, B. Hess, and the GROMACS development team, GROMACS User Manual version 5.1.2, online at www.gromacs.org.
[19] J. M. Martínez and L. Martínez, J. Comput. Chem. 24, 819 (2003).

[20] L. Martínez, R. Andrade, E. G. Birgin, and J. M. Martínez, J. Comput. Chem. 30, 2157 (2009).

[21] P. Deift and X. Zhou, Ann. Math. 137, 295 (1993).

[22] H. J. C. Berendsen, J. P. M. Postma, W. F. van Gunsteren, A. DiNola, and J. R. Haak, J. Chem. Phys. 81, 3684 (1984).

[23] S. Nosé, J. Chem. Phys. 81, 511 (1984).

[24] W. G. Hoover, Phys. Rev. A 31, 1695 (1985).

[25] M. Parrinello and A. Rahman, Phys. Rev. Lett. 45, 1196 (1980).

[26] U. Essmann, L. Perera, M. L. Berkowitz, T. Darden, H. Lee, and L. G. Pedersen, J. Chem. Phys. 103, 8577 (1995).

[27] R. W. Hockney, Methods Comput. Phys. 9, 135 (1970).

[28] B. Hess, H. Bekker, H. J. C. Berendsen, and J. G. E. M. Fraaije, J. Comput. Chem. 18, 1463 (1997).

[29] M. Yamaguchi, M. Takahashi, F. Harusawa, and S. Fukushima, J. Soc. Cosmet. Chem. Jpn. 12, 16 (1978).

[30] S. E. Feller and R. W. Pastor, J. Chem. Phys. 111, 1281 (1999).

[31] S. J. Marrink, A. H. de Vries, and A. E. Mark, J. Phys. Chem. B 108, 750 (2004).

[32] W. K. den Otter, J. Chem. Phys. 123, 214906 (2005).

[33] F. J. van Eerden, D. H. de Jong, A. H. de Vries, T. A. Wassenaar, and S. J. Marrink, Biochim. Biophys. Acta (BBA) Biomembranes 1848, 1319 (2015).

[34] Y. Guo, V. A. Baulin, and F. Thalmann, Soft Matter 12, 263 (2016).

[35] J. F. Nagle and S. Tristram-Nagle, Biochim. Biophys. Acta (BBA) - Rev. Biomembranes 1469, 159 (2000).

[36] T. Heimburg, Thermal Biophysics of Membranes (Wiley-VCH, Weinheim, 2007).

[37] W.-G. Seo, D. Zhou, and F. Tsukihashi, Mater. Trans. 46, 643 (2005).

[38] D. Marsh, Handbook of Lipid Bilayers, 2nd ed. (CRC, Boca Raton, FL, 2013). 